NISSUNA UMANA INVESTIGAZIONE SI PUO DIMANDARE VERA SCIENZIA S'ESSA NON PASSA PER LE MATEMATICHE DIMOSTRAZIONI LEONARDO DA VINCI

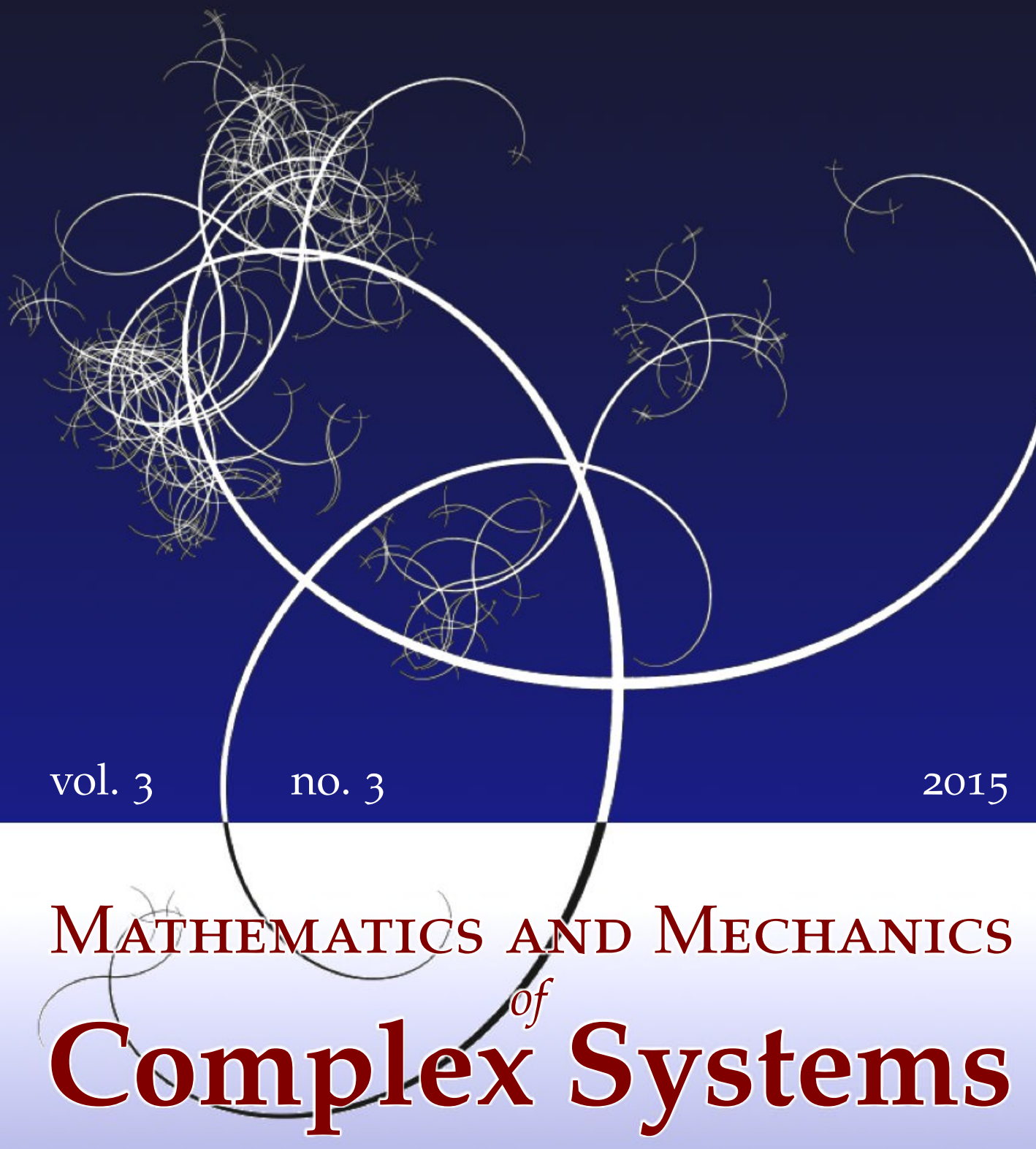

SANICHIRO YOSHIDA

COMPREHENSIVE DESCRIPTION OF DEFORMATION OF SOLIDS AS WAVE DYNAMICS 


\title{
COMPREHENSIVE DESCRIPTION OF DEFORMATION OF SOLIDS AS WAVE DYNAMICS
}

\author{
SANICHIRO YOSHIDA
}

\begin{abstract}
Deformation and fracture of solids are discussed as comprehensive dynamics based on a field theory. Applying the principle of local symmetry to the law of elasticity and using the Lagrangian formalism, this theory derives field equations that govern dynamics of all stages of deformation and fracture on the same theoretical foundation. Formulaically, these field equations are analogous to the Maxwell equations of electrodynamics, yielding wave solutions. Different stages of deformation are characterized by differences in the restoring mechanisms responsible for the oscillatory nature of the wave dynamics. Elastic deformation is characterized by normal restoring force generating longitudinal waves; plastic deformation is characterized by shear restoring force and normal energy-dissipative force generating transverse, decaying waves. Fracture is characterized by the final stage of plastic deformation where the solid has lost both restoring and energy-dissipative force mechanisms. In the transitional stage from the elastic regime to the plastic regime where both restoring and energydissipative normal force mechanisms are active, the wave can take the form of a solitary wave. Experimental observations of transverse, decaying waves and solitary waves are presented and discussed based on the field theory.
\end{abstract}

\section{Introduction}

Most conventional approaches classify deformation of solids into separate regimes and discuss its mechanics based on the constitutive relation of each regime. The elastic and plastic regimes are defined, respectively, as the regimes where the constitutive relation is characterized by linear and nonlinear stress-strain relations, and fracture is considered as an independent phenomenon where a preexisting crack grows by itself. For each regime, a specific theory is used: continuum mechanics [Spencer 1980; Landau and Lifshitz 1986] for the elastic regime, various theories of plasticity [Hill 1998; Lubliner 2008] for the plastic regime and fracture mechanics [Griffith 1921; Irwin 1948] for the fracturing regime. When an external load is applied to a solid object and the resultant deformation is viewed as the response

\section{Communicated by Francesco dell'Isola.}

PACS2010: 46.

Keywords: deformation of solids, plastic deformation transverse-wave, elasto-plastic solitary-wave. 
of the entire object, it is true that the deformation mechanics undergone in these regimes exhibits the stress-strain characteristics of the respective regimes. If the same object is analyzed in a local region, however, it is obvious that the deformation status can be different from other local regions, and hence, on the global level, the deformation should be characterized by multiple constitutive relations at the same time. A metal specimen freshly taken out of an annealing oven has a number of dislocations; as soon as an external load is applied to such a specimen, the dislocations are activated causing local plastic deformation. These local nonlinear behaviors are not observed in the stress-strain characteristic of the entire specimen as most of the specimen undergoes purely elastic deformation at this stage. At the other extreme, a metal specimen about to fail recovers from the deformed state if the load is removed. It is unrealistic to rely on a regime-specific theory in any stage of deformation. It is essential to use a theory that can describe all stages of deformation comprehensively.

Comprehensive theory of deformation and fracture is not only useful to describe the situation where elastic and plastic deformations coexist. It is also essential to formulate the transitions from one regime to another. Generally, the life of solids under external loads is a progression from elastic deformation to fracture through plastic deformation. In the tensile or compression mode of deformation where the stress increases with the passage of time, the deformation exhibits this pattern of progression as a function of the increasing stress. Even in the fatigue mode of deformation where the magnitude of the external load remains the same, most solids follow the same pattern of progression [Ichinose et al. 2006]. In engineering, often analysis of the transitional stage from one regime to the next is more important than analysis within a certain regime. If the remaining life of a machine part is known, it becomes unnecessary to replace it at an earlier stage, contributing to the conservation of natural resources. To analyze these transitional stages, the theory must be regime-independent. Furthermore, these transitions involve multiple scale levels. Fracture of solids is always initiated at the atomistic scale and evolves to the microscopic scale and eventually to the macroscopic scale; defects of a size comparable to several atoms grow to the microscopic level and eventually to the macroscopic level when the entire specimen fails. A universal approach is essential.

In this regard, a recent field theory of deformation and fracture has an advantage [Yoshida 2015]. Requesting local symmetry [Aitchison and Hey 1989] in Hooke's law, this theory derives field equations that govern the displacement field of solids under deformation. Formulaically, the field equations are very similar to Maxwell's equations of electrodynamics. From a dynamical point of view, the field equations represent synergetic interaction between the translational and rotational modes of displacement. This interaction can be interpreted as Lenz's law 
analogous to Faraday's law in electrodynamics. In the present context, Lenz's law represents solids' response to reduce the disturbance caused by an external load. The general solution to the field equations is a wave function where the form of the wave characteristics depends on the regime. The elastic regime can be characterized by a longitudinal wave known as the wave of compression and a rotational wave known as the wave of deformation. In this regime, the elasticity is a longitudinal effect where the solid material responds to the force due to an external load elastically. The plastic regime is characterized by a transverse wave that decays due to the irreversibility of plasticity. In this regime, the elasticity is a transverse effect due to differential rotation; the solid material responds elastically to the external torque and not to the force. The irreversibility is due to the energy dissipation associated with irreversible motion of localized normal $\operatorname{strain}^{1}$ in the direction of the local velocity. Thus, the longitudinal effect is energy-dissipative. The solid material resists the external force energy-dissipatively and the external torque elastically. Fracture is the stage of deformation where the solid material has lost all the mechanisms to resist the external load, elastically or energy-dissipatively, and the only reaction to the load is to create discontinuity. Under some conditions, the energy-carrying wave takes the form of a solitary wave. In this situation, the solid material does not exhibit resistive force, and the stress-strain curve plateaus. A similar phenomenon occurs in the transitional stage from the plastic to fracture regimes. The material dissipates energy from the external load via propagation of a solitary wave. When the solitary wave stops moving, the material loses the energydissipative mechanism completely, and it fractures. Thus, the transition from one regime of deformation to another can be identified as a change in the way the solid material responds to an external load and characterized as different forms in the displacement wave.

The similarity between electrodynamics and the present field deformation theory is not limited to the formulaic resemblance in the field equations. As mentioned above, the energy dissipation in plastic deformation is caused by motion of localized normal strain due to the local velocity field. From the field-theoretical viewpoint, the normal strain can be interpreted as the charge of symmetry associated with the local symmetry of Hooke's law. From the viewpoint of Lagrangian dynamics, the normal strain can be interpreted as representing the Noether current associated with the invariance of the corresponding Lagrangian density. From this standpoint, this quantity can be identified as corresponding to the electric charge and called the deformation charge. Note that the electric charge is proportional to divergence of the electric field and the normal strain is divergence of the displacement field. With this interpretation, the energy dissipation in plastic deformation

\footnotetext{
${ }^{1}$ Strictly speaking, it is the rate of normal strain or the temporal derivative of normal strain. For simplicity, it is called the normal strain.
} 
can be understood as a phenomenon analogous to Ohmic loss in electrodynamics where the electromagnetic field loses energy when a free electric charge is moved by the electric field. From the perspective of energy flow, the transverse displacement wave carries elastic energy through the material and the charge flow dissipates the energy. When the transverse wave decays completely and the charge stops moving, the material loses all mechanisms to transfer the work provided by the external load from one side of the specimen to the other or dissipate it. This is when the material fractures. The situation is analogous to electrical breakdown of dielectric media [Yoshida 2000]. When the level of ionization is low and hence the density of free charge is low, the propagation of electromagnetic waves is the dominant mechanism of energy flow. When the conduction current density increases to a certain level, the Ohmic loss becomes the dominant mechanism to dissipate the energy provided by the external circuit. Eventually, the current density becomes infinitely high, and that is when the medium is electrically broken.

A number of authors have formulated nonlinear behavior of deformation. Among them, the following models have been proposed as useful tools for unified description of elastic and plastic deformations and are worth mentioning here. These models are based on the framework established by Toupin [1964] and Mindlin [1965] and known as Toupin-Mindlin strain-gradient theory. This theory postulates that the strain energy depends both on the symmetric strain tensor and the second gradient of displacement. By introducing a Lagrangian action both in the material and the spatial description, Auffray et al. [2015] have formulated a material description for second-gradient continua. Javili et al. [2013] have generalized the work by Mindlin [1965] and formulated a geometrically nonlinear theory of higher-gradient elasticity accounting for boundary energies. By means of the least action principle, Madeo et al. [2013] have derived a general set of equations of motion and duality conditions to be imposed at macroscopic surfaces of discontinuity in partially saturated, solid second-gradient porous media. Fleck and Hutchinson [1997] have applied the Toupin-Mindlin strain-gradient framework to plastic deformation and proposed phenomenological theories of strain-gradient plasticity.

Connections between these formalisms based on the strain-gradient theory and the present field theory are not straightforward and are not fully understood at this point. Nevertheless, it is worth pointing out some similarities and a contrast between these formalisms and the present theory. As will be discussed in the next section, the present theory incorporates nonlinearity by allowing the transformation matrix of linear elasticity, known as the displacement gradient tensor, to be coordinate-dependent. The components of the displacement gradient tensor are strain, which is essentially the first gradient of displacement. Thus, the fact that we allow its coordinate dependence means that we automatically consider the second gradient of displacement. Naively speaking, this corresponds to the 
approach taken by the strain-gradient theory where the second gradient of displacement is included in the expression of the strain energy. Auffray et al. [2015] and Madeo et al. [2013] apply the Lagrangian formalism to deduce the evolution equations. This procedure seems analogous to the present theory whereby the field equations are derived through the application of the Lagrangian formalism to the gauge potential. Moreover, the use of both the material and spatial descriptions in the Lagrangian action made by Auffray et al. indicates that the two descriptions interact with each other in the same or similar fashion as the gauge field and the linear elastic displacement field interact with each other in the present theory.

As for the contrast, the following point should be noted. In the present field theory, the nonlinearity is introduced in conjunction with the coordinate dependence of the transformation matrix that describes the local linear elasticity (the base theory). In other words, the nonlinearity is associated with the curvilinearity of the coordinate axes and not intrinsic in the base theory. In the case of the strain-gradient theory, on the other hand, the starting Lagrangian encompasses nonlinearity as the strain energy expression has the term containing the second gradient of displacement; thus, the formalism derived from the strain-gradient theory is intrinsically able to describe the nonlinear nature of geometry such as the inclusion of porosity and layered structures. It may be possible to incorporate these geometrical nonlinear effects by the use of an appropriate compensation field, but the possibility is not clear at this time. It is safe to assume, at least for now, that the present theory is applicable to the cases where the local deformation can be modeled to obey the law of linear elasticity.

The aim of this paper is to provide an overview of this field-theoretical approach to dynamics of deformation and fracture. After briefly reviewing the concept of local symmetry on which the present theory is based, the field equations will be derived. Through physical interpretations of the field equations, deformation dynamics will be discussed from the viewpoint of force acting on a unit volume of the solid material. It will be shown that the transverse (shear) force is restoring regardless of the regime whereas the longitudinal (normal) force is restoring in the elastic regime and energy-dissipative in the plastic regime. It will also be shown that these restoring forces cause longitudinal and transverse wave nature of elastic and plastic deformation and solitary wave nature in the transitional stage from the elastic regime to the plastic regime. The wave equations for the respective regimes will be derived from the field equations and will be solved analytically under some conditions. The energy-dissipative nature of the dynamics will be explained through the concept of deformation charge. Supporting experimental results will be presented to discuss these wave characteristics and dynamics of the deformation charge. 


\section{Theory}

Details of the present field theory can be found elsewhere [Yoshida 2015]. In short, this field theory formulates deformation dynamics based on two postulates. The first postulate is that a solid of any deformation status locally obeys the law of linear elasticity (Hooke's law). The local region that obeys Hooke's law is referred to as the deformation structural element. The second postulate is that, as long as the solid remains a continuum, all the deformation structural elements of the object are logically connected by a field known as the gauge field. The first postulate is rationalized through the consideration that regardless of the stage of deformation it is always possible to find a local region where the interatomic potential is approximated by a quadratic function of the displacement of the atom from its equilibrium position or, equivalently speaking, the field force on the atom is elastic force whose magnitude is proportional to the displacement. The above-mentioned claim that a solid object about to fail recovers from the deformed state to a certain extent if the load is removed is an example. This postulate indicates that each of these local elastic deformations can be expressed by a transformation that represents Hooke's law with the local coordinate system (local frame). If the local frame is oriented to the principal axis, the corresponding transformation matrix is diagonal as the shear components of the stress and strain matrices are all zero. Here, it is important to note that the principal axes of the deformation structural elements within the same object do not necessarily have the same orientation. In fact, it is usually the case that in the plastic regime they are oriented randomly, as will be discussed later. This means that we cannot define a principal axis with the global coordinate system (global frame) and that therefore we cannot express the local elastic deformations inclusively. This is where the second postulate comes into the picture.

The second postulate can be argued in various ways based on the principle of local symmetry. The most intuitive argument will be as follows. The situation where multiple deformation structural elements undergo linear elastic deformation with the respective principal axes raises a question: "are the local elastic deformations expressed in the respective local coordinates independent of one another?" or, equivalently, "do the deformation structural elements know one another's elastic deformation?" The answer must be: "they are not independent of one another" or "they should know one another's behavior". Otherwise, the situation becomes the same as the same number of independent solid objects (not connected with one another) as the deformation structural elements experience elastic deformation independently. Then the next question will be: "how are they connected?" We can find a short answer to this question by recalling that we try to express all the local elastic deformations inclusively with the global coordinates under the situation where the local elastic deformations have their own orientations of the 


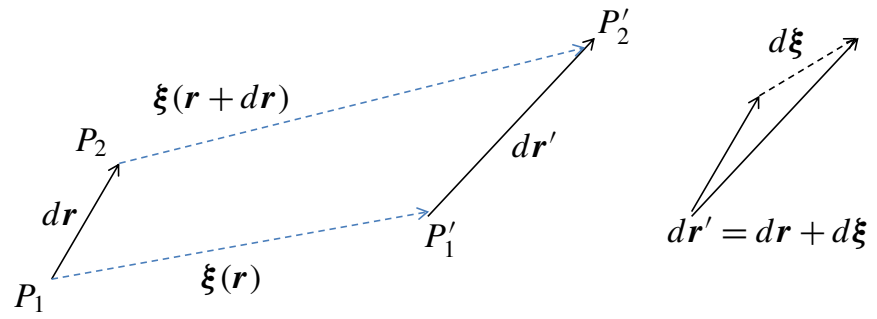

Figure 1. Infinitesimal line element changes its length under deformation.

principal axis. In other words, each deformation structural element experiences stretch and compression along mutually different orientations. In order to express these behaviors inclusively in the global frame, it is necessary to align all these axes in the same orientation. This argument indicates that the gauge field has something to do with rotational dynamics. As will be discussed later in this paper, indeed the present gauge field is associated with rotational dynamics. From the field-theoretical viewpoint, the gauge field compensates for the fact that the elastic deformation expressed in the global coordinate does not consider the nonlinearity of the dynamics. From this standpoint, this field is referred to as the compensation field. The portion of the dynamics that we overlook by artificially aligning the deformation structural elements, which is responsible for the nonlinearity and the irreversibility of plastic deformation, is crammed into the potential generated by the gauge field. Naturally, this potential is rotation-like. We say that the gauge field makes the law of linear elasticity locally symmetric. Mathematically, it can be stated as follows. Under the situation where deformation structural elements undergo respective elastic deformation, the transformation matrix is coordinatedependent. Consequently, the associated physics law cannot be written with the global coordinates in the same form as the local coordinates. This is because the expression of the physics law involves differentiation and the coordinate dependence of the transformation matrix generates the extra term resulting from the differentiation of the matrix. The gauge field regains the formality in the global frame by adding an extra term (the gauge term) to the usual derivatives so that this term cancels out the effect of the differentiation of the transformation matrix. The derivative with the gauge term is referred to as the covariant derivative. It is interesting to note that mathematicians call the gauge field the connection field. In the present case, the gauge field literally connects deformation structural elements so that they form a single continuum.

2.1. Deformation as linear transformation. Consider in Figure 1 a solid object under deformation. By this deformation, an infinitesimal line element vector $\boldsymbol{\eta}=$ $(d x, d y, d z)$ changes its length and direction by coordinate-dependent displacement vector $\boldsymbol{\xi}(x, y, z)$. Expressing the resultant line element vector as $\boldsymbol{\eta}^{\prime}$, we can 
express the deformation with the linear transformation

$$
\boldsymbol{\eta}^{\prime}=(I+\beta) \equiv U \boldsymbol{\eta},
$$

where $I$ is the unit matrix and $\beta$ is the displacement gradient tensor

$$
\beta=\left(\delta_{i j}+\frac{\partial \xi_{i}}{\partial x^{j}}\right) .
$$

Here, $\xi$ is the displacement vector. In the theory of elasticity, the elastic force is proportional to the stretch or the differential displacement $d \xi$. Therefore, for the theory to be invariant, the differential displacement vector must be transformed in the same fashion as the displacement vector itself. Otherwise, after the transformation, the elastic force law cannot be written in the same form as before the transformation. This means that, after the transformation, the differential displacement must have the same form as the transformation of the differential displacement. If we use the usual differentiation, apparently this is not the case as the following expression indicates:

$$
d(U \boldsymbol{\xi})=d U \boldsymbol{\xi}+U d \boldsymbol{\xi}
$$

The condition $d(U \boldsymbol{\xi})=U d \xi$ holds only when $d U=0$ or the transformation is coordinate-independent. Thus, it becomes necessary to replace the usual derivatives with covariant derivatives or introduce a gauge term $\Gamma_{i}$ :

$$
D_{i}=\frac{\partial}{\partial x_{i}}-\Gamma_{i} \equiv \partial_{i}-\Gamma_{i} .
$$

It is easily proved that, if the gauge term transforms as (5), the differential after the transformation has the same form as the transformation of the differential, that is, $D_{i}^{\prime}(U \xi)=U\left(D_{i} \xi\right)$ :

$$
\Gamma_{i}^{\prime}=U \Gamma_{i} U^{-1}+\frac{\partial U}{\partial x^{i}} U^{-1} .
$$

Here the prime' indicates the quantity after the transformation.

Now consider the physical meaning of the gauge term:

$$
D \xi_{i}=\left(\frac{\partial \xi_{i}}{\partial x}-\Gamma_{x} \xi_{i}\right) d x+\left(\frac{\partial \xi_{i}}{\partial y}-\Gamma_{y} \xi_{i}\right) d y+\left(\frac{\partial \xi_{i}}{\partial z}-\Gamma_{z} \xi_{i}\right) d z \equiv d \xi_{i}-A_{i}
$$

In elastic deformation, the rotation matrix represents rigid body rotation of the material, which does not involve length change. In (6), the actual change in the length of the displacement vector is all in $d \xi_{i}$. Thus, $A_{i}$ can be interpreted as representing a displacement vector that rotates the deformation structural element so that the differential displacement vector contains only the change due to physically true deformation and not to the geometrical effect. Figure 2 illustrates this situation 

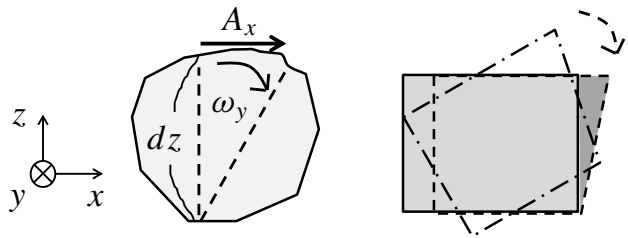

Figure 2. Vector potential as displacement vector to align deformation structural element.

schematically. Here $\omega_{y}$ represents the rotation that must be removed to find out the true deformation.

The above argument that the vector potential represents material rotation associated with the covariant derivatives can be justified from the following viewpoint. In the theory of elasticity, differential displacement (deformation gradient tensor) can be separated into the symmetric and asymmetric portions referred to as the strain and rotation matrices, respectively. When the coordinate axes are chosen to be the principal axes, the strain matrix is diagonal, or its shear components are all zero. Now consider that different parts of a given solid object undergo their respective elastic deformations. As an example, when an initially elastic object has a defect, the four blocks will undergo different elastic deformation as Figure 3 illustrates schematically. Under this condition, the four blocks have their own principal axes. It becomes impossible to describe the four elastic deformations with a common principal axis.

Based on the above interpretation, the explicit form of the spatial parts of vector potential $\boldsymbol{A}$ can be identified as

$$
\boldsymbol{A}=\left(\begin{array}{rcr}
0 & -\omega_{z} & \omega_{y} \\
\omega_{z} & 0 & -\omega_{x} \\
-\omega_{y} & \omega_{x} & 0
\end{array}\right)\left(\begin{array}{l}
d x \\
d y \\
d z
\end{array}\right)=\left(\begin{array}{r}
-\omega_{z} d y+\omega_{y} d z \\
\omega_{z} d x-\omega_{x} d z \\
-\omega_{y} d x+\omega_{x} d y
\end{array}\right)
$$

The temporal component of vector potential $\boldsymbol{A}$ can be understood in conjunction with the temporal differentiation as follows. Suppose deformation dynamics $\psi$

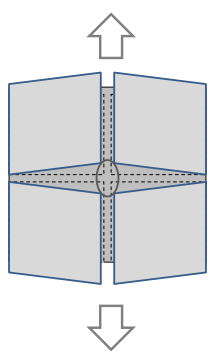

Figure 3. Local region containing a defect at the center. 

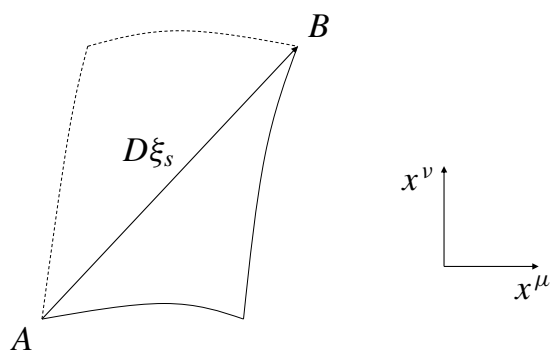

Figure 4. Gauge field strength for nonlinear deformation.

propagates as a wave. In general, the wave function can be put in the form

$$
\psi=f\left(\omega_{0} t-\boldsymbol{k} \cdot \boldsymbol{r}\right)=f\left(\omega_{0} t-k(\alpha x+\beta y+\gamma z)\right),
$$

where $\omega_{0}$ is the angular frequency, $\boldsymbol{k}$ is the propagation vector and $\alpha, \beta$ and $\gamma$ are directional cosines. Expressing the derivative of function $f$ as $f^{\prime}$, we find

$$
f^{\prime}=\frac{1}{\omega_{0}} \frac{\partial \psi}{\partial t}=-\frac{1}{k}(\nabla \psi) \cdot \hat{k},
$$

where $\hat{k}$ is the unit vector of $\boldsymbol{k}$. Noting the ratio of the angular frequency to the propagation vector is the phase velocity $c, \omega_{0} / k=c$, we can rewrite (9) as ${ }^{2}$

$$
\frac{\partial \psi}{\partial t}=-(\nabla \psi) \cdot c \hat{k}=-(\nabla \psi) \cdot \boldsymbol{c} .
$$

The spatial and temporal components of vector potential $\boldsymbol{A}$ are to compensate the spatial and temporal differentiations. Thus, they can be interpreted as being corresponding to terms $(\nabla \psi)$ and $\partial \psi / \partial t$ in (10). This interpretation leads to the vector potential expression of $\boldsymbol{A}$ as follows. Intuitively, the temporal component of the vector potential can be interpreted as representing the same effect as the spatial component explained in Figure 2; it represents the effect of the compensating potential in the time domain wherein the dynamics is a wave phenomenon traveling at the phase velocity $c$ :

$$
A^{\mu}=\left(A^{0}, A^{1}, A^{2}, A^{3}\right)=\left(\frac{\phi^{0}}{c}, A^{1}, A^{2}, A^{3}\right) .
$$

Now consider how vector potential $\boldsymbol{A}$ acts on the deformation dynamics. We know that $\boldsymbol{A}$ represents the dynamics that local linear elastic dynamics cannot represent. In other words, it accounts for the compensation we need to pay as the penalty for pretending that the dynamics is linear elastic. This effect can be formulated by

\footnotetext{
${ }^{2}$ If we repeat the same procedure to find the secondary derivatives, we will obtain the elastic wave equation where the phase velocity is the square root of the ratio of the elastic modulus to the density.
} 
comparing clockwise and counterclockwise covariant derivatives. Figure 4 illustrates the clockwise and counterclockwise differentiation schematically. Dropping the second-order differentials, the clockwise case is

$$
D_{\mu}\left(D_{\nu} \xi_{s} d x^{\nu}\right) d x^{\mu}=\partial_{\mu} \partial_{\nu} \xi_{s} d x^{\nu} d x^{\mu}-\partial_{\mu}\left(\Gamma_{\nu} \xi_{s} d^{\nu}\right) d x^{\mu}+\Gamma_{\mu} \Gamma_{\nu} \xi_{s} d x^{\nu} d x^{\mu} .
$$

Here from the definition, $\Gamma_{\nu} \xi_{s} d x^{\nu}$ can be interpreted as $A_{\nu}$, and so

$$
D_{\mu}\left(D_{\nu} \xi_{s} d x^{\nu}\right) d x^{\mu}=\partial_{\mu} \partial_{\nu} \xi_{s} d x^{\nu} d x^{\mu}-\partial_{\mu} A_{\nu} d x^{\mu}+\frac{1}{\xi_{s}} A_{\mu} A_{\nu} .
$$

The counterclockwise case can be expressed with the vector potential in the same fashion. Thus, the difference between the clockwise and counterclockwise cases is

$$
\begin{aligned}
D_{\mu}\left(D_{\nu} \xi_{s} d x^{\nu}\right) d x^{\mu}-D_{\nu}\left(D_{\mu} \xi_{s} d x^{\mu}\right) d x^{\nu} & \\
& =\left(\partial_{\nu} A_{\mu} d x^{\nu}-\partial_{\mu} A_{\nu} d x^{\mu}\right)+\frac{1}{\xi_{s}}\left[A_{\mu}, A_{\nu}\right] .
\end{aligned}
$$

In the infinitesimal limit, $d x^{\nu}=d x^{\mu}=d s$, and division of the above equation by $d s$ leads to

$$
\left[D_{\mu}, D\right]_{s} \xi_{s} d s=\left(\partial_{\nu} A_{\mu}-\partial_{\mu} A_{\nu}\right)+\frac{1}{\xi_{s} d s}\left[A_{\mu}, A_{\nu}\right] \equiv F_{\mu \nu} .
$$

Here $F_{\mu \nu}$ is known as the field stress tensor. Each component of vector potential equation (11) represents a displacement component. It is easily proved that they are commutable; hence, the $\left[A_{\mu}, A_{\nu}\right]$ term of (15) is zero. With this, we obtain the explicit form

$$
F_{\mu \nu}=\left(\begin{array}{cccc}
0 & -v^{1} / c & -v^{2} / c & -v^{3} / c \\
v^{1} / c & 0 & -\omega^{3} & \omega^{2} \\
v^{2} / c & \omega^{3} & 0 & -\omega^{1} \\
v^{3} / c & -\omega^{2} & \omega^{1} & 0
\end{array}\right) .
$$

Here $v^{i}, i=1,2,3$, is the time derivative of $A^{i}$ and $\omega^{i}, i=1,2,3$, is the rotation associated with the corresponding components of the displacement due to vector potential $\boldsymbol{A}$ :

$$
\omega^{k}=\frac{\partial A^{j}}{\partial x^{i}}-\frac{\partial A^{i}}{\partial x^{j}}
$$

2.2. Lagrangian and field equation. The field stress tensor is not invariant under the transformation $U$. However, it is easily proved that the trace $F_{\mu \nu} F^{\mu \nu}$ is invariant [Yoshida 2011]. This indicates that we can construct the Lagrangian of free particles (without the interaction with the gauge field or vector potential) in the form proportional to $F_{\mu \nu} F^{\mu \nu}$. Knowing that the phase velocity $c_{\text {shear }}$ associated with shear force has the form

$$
c_{\text {shear }}=\sqrt{G / \rho},
$$


where $G$ is the shear modulus and $\rho$ is the density, and that the Lagrangian is kinetic energy minus potential energy, we can identify the Lagrangian density as

$$
L=-\frac{G}{4} F_{\mu \nu} F^{\mu \nu}=\frac{\rho v^{2}}{2}-\frac{G \omega^{2}}{2} .
$$

Here the first term is the kinetic energy of the unit volume and the second term is the rotational spring potential energy. The Lagrangian in the form of (19) indicates the phase velocity $c$ in (16) is in fact the shear wave velocity (18). By adding the interaction term, we can identify the full Lagrangian in the form

$$
L=-\frac{G}{4} F_{\mu \nu} F^{\mu \nu}+G j^{\mu} A_{\mu}=\frac{\rho v^{2}}{2}-\frac{G \omega^{2}}{2}+\frac{G}{c} j^{0} A_{0}+G j^{i} A_{i} .
$$

Here $j^{0}$ and $j^{i}$ are the temporal and spatial components of the quantity known as the charge of symmetry, and they are connected with the phase velocity (18) as

$$
j^{\mu}=\left(\frac{j^{0}}{c}, j^{1}, j^{2}, j^{3}\right) .
$$

The four vector $j^{\mu}$ describes how the material interacts with the gauge field and is conserved under the governing transformation (in this case transformation $U$ ).

With this Lagrangian, the Euler-Lagrangian equation of motion associated with $A_{\mu}$ can be given as

$$
\partial_{\nu} \frac{\partial L}{\partial\left(\partial_{\nu} A_{\mu}\right)}-\frac{\partial L}{\partial A_{\mu}}=0 .
$$

This leads to the following field equations:

$$
\begin{aligned}
\nabla \cdot \boldsymbol{v} & =-j_{0}, \\
\nabla \times \boldsymbol{v} & =\frac{\partial \boldsymbol{\omega}}{\partial t}, \\
\nabla \times \boldsymbol{\omega} & =-\frac{1}{c^{2}} \frac{\partial \boldsymbol{v}}{\partial t}-\boldsymbol{j}, \\
\nabla \cdot \boldsymbol{\omega} & =0 .
\end{aligned}
$$

Here $c$ appearing on the right-hand side of (25) is the phase velocity (18)

2.3. Deformation charge and comprehensive description. Rearranging the terms, we can put the field equation (25) in the form [Yoshida 2011; 2008]

$$
\frac{1}{c^{2}} \frac{\partial \boldsymbol{v}}{\partial t}=-\nabla \times \boldsymbol{\omega}-\boldsymbol{j}
$$

The spatial component of the charge $j^{\mu}$ appearing on the right-hand side of the third field equation (25) represents the longitudinal effect of the gauge field on the material and is very important to describe the dynamics. The $c$ appearing in the first 


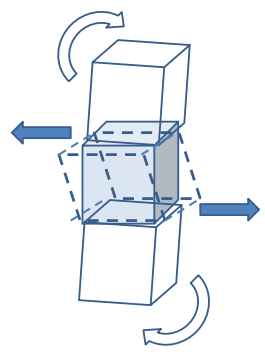

Figure 5. Shear force due to differential rotational displacement.

term on the right-hand side of the same equation represents the phase velocity (18), the velocity that the spatial pattern of the displacement field propagates. Using the phase velocity expression (18) and multiplying both sides of this equation by $\rho$, we can put (27) in the form

$$
\rho \frac{\partial \boldsymbol{v}}{\partial t}=-G \nabla \times \boldsymbol{\omega}-G \boldsymbol{j} .
$$

The left-hand side of (28) is found to have the form of the product of the mass and acceleration of the unit volume. Thus, according to Newton's second law, the right-hand side of (27) is the external force acting on the unit volume. Here the first term $G \nabla \times \omega$ represents the shear force exerted by the neighboring blocks of the material due to their differential rotations, and the second term $G \boldsymbol{j}$ can be identified as the longitudinal force density. Figure 5 illustrates the shear force schematically. The form of this second term differentiates different regimes of deformation, as will be discussed below.

Elastic regime. Take the divergence of both sides of (25) and substitute the resultant $\nabla \cdot \boldsymbol{v}$ with (23). This provides us with an equation of continuity associated with the conservation of charge $j_{0}=-\nabla \cdot \boldsymbol{v}$ :

$$
\rho \frac{\partial(\nabla \cdot \boldsymbol{v})}{\partial t}=-\nabla \cdot(G \boldsymbol{j}) .
$$

Using $\partial \boldsymbol{\xi} / \partial t=\boldsymbol{v}$, rewrite the left-hand side of (29) as

$$
\rho \frac{\partial^{2}(\nabla \cdot \boldsymbol{\xi})}{\partial t^{2}}=-\nabla \cdot(G \boldsymbol{j})
$$

The quantity $\nabla \cdot \xi$ appearing on the left-hand side of (30) is known as the volume expansion in continuum mechanics. With the interpretation that $G \boldsymbol{j}$ represents the longitudinal force, (30) can be interpreted as the equation of motion of a unit volume experiencing volume expansion due to differential longitudinal (normal) force. We can identify the explicit form of $G \boldsymbol{j}$ for an isotropic elastic medium based on continuum mechanics. 
Recall that the constitutive relation can be written in the following form based on Cauchy's formalism:

$$
\left(\begin{array}{c}
\sigma_{x x} \\
\sigma_{y y} \\
\sigma_{z z} \\
\sigma_{x y} \\
\sigma_{y z} \\
\sigma_{z x}
\end{array}\right)=\left(\begin{array}{cccccc}
\lambda+2 G & \lambda & \lambda & 0 & 0 & 0 \\
\lambda & \lambda+2 G & \lambda & 0 & 0 & 0 \\
\lambda & \lambda & \lambda+2 G & 0 & 0 & 0 \\
0 & 0 & 0 & 2 G & 0 & 0 \\
0 & 0 & 0 & 0 & 2 G & 0 \\
0 & 0 & 0 & 0 & 0 & 2 G
\end{array}\right)\left(\begin{array}{c}
\epsilon_{x x} \\
\epsilon_{y y} \\
\epsilon_{z z} \\
\epsilon_{x y} \\
\epsilon_{y z} \\
\epsilon_{z x}
\end{array}\right),
$$

where $\sigma_{i j}$ denotes the $j$-th component of the stress acting on plane $i, \lambda$ is the first Lamé coefficient and $\epsilon_{i j}$ is the strain defined as

$$
\epsilon_{i j}=\frac{1}{2}\left(\frac{\partial \xi_{j}}{\partial x_{i}}+\frac{\partial \xi_{i}}{\partial x_{j}}\right)
$$

Considering the $x$ component of the net external force acting on a cube of unit volume, we obtain the equation of motion

$$
\rho \frac{\partial^{2} \xi_{x}}{\partial t^{2}}=\frac{\partial \sigma_{x x}}{\partial x}+\frac{\partial \sigma_{y x}}{\partial y}+\frac{\partial \sigma_{z x}}{\partial z} .
$$

Substituting the corresponding stress tensor components of the constitutive equation (31) into the right-hand side and using (32), we can rewrite

$$
\rho \frac{\partial^{2} \xi_{x}}{\partial t^{2}}=G \nabla^{2} \xi_{x}+\frac{\partial}{\partial x}(\lambda+G) \nabla \cdot \xi
$$

View $G \nabla^{2} \xi_{x}$ as the $x$ component of $G \nabla^{2} \xi$, and use the mathematical identity $\nabla \times \nabla \times=\nabla(\nabla \cdot)-\nabla^{2}$ to rewrite $G \nabla^{2} \xi$ as

$$
G \nabla^{2} \boldsymbol{\xi}=-G \nabla \times \nabla \times \xi+G \nabla(\nabla \cdot \xi) .
$$

On the right-hand side of (35), the longitudinal effect is represented by the second term. Taking only this term and noting that the $x$ component of $\nabla(\nabla \cdot \xi)$ can be put as $(\nabla(\nabla \cdot \xi))_{x}=\partial(\nabla \cdot \xi) / \partial x$, we can rewrite (34) as

$$
\rho \frac{\partial^{2} \xi_{x}}{\partial t^{2}}=G(\nabla(\nabla \cdot \xi))_{x}+\frac{\partial}{\partial x}(\lambda+G)(\nabla \cdot \xi)=\frac{\partial}{\partial x}(\lambda+2 G)(\nabla \cdot \xi) .
$$

Including the $y$ and $z$ components, we can express the longitudinal force vector appearing on the right-hand side of (28) in the form

$$
G \boldsymbol{j}=-\nabla(\lambda+2 G)(\nabla \cdot \xi) .
$$

We can identify this form of $G \boldsymbol{j}$ as the longitudinal force in the elastic case. Here $(\lambda+2 G)(\nabla \cdot \xi)$ is the longitudinal force at a surface of the unit-volume cube proportional to the strain at that point, and the entire right-hand side represents 


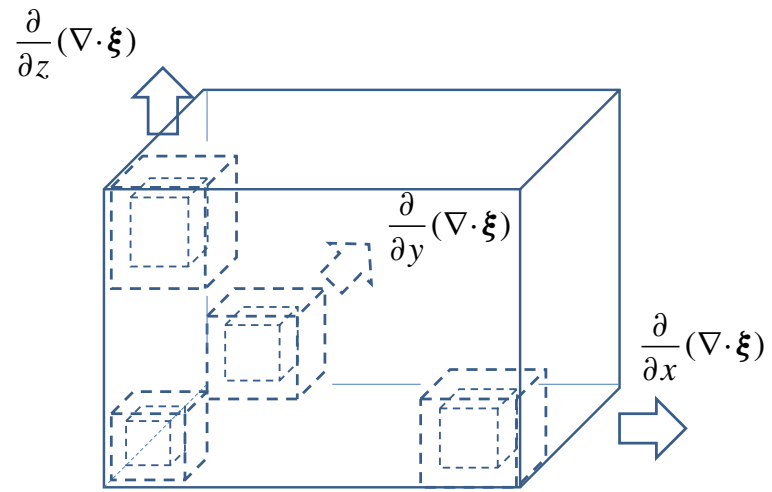

Figure 6. Elastic force proportional to volume expansion.

the differential longitudinal force, the difference of $(\lambda+2 G)(\nabla \cdot \xi)$ between the leading and tailing surfaces of the unit-volume cube. Figure 6 illustrates the force schematically.

Note that the first term on the right-hand side of (35) represents the shear force and can be written as $\nabla \times \nabla \times \xi=\nabla \times \omega$. This terms appears as the shear force term in (28). In the elastic limit, (35), hence field equation (25), reduces to Cauchy's constitutive equation.

From the viewpoint of the equation of continuity, (29) indicates that compression or rarefaction of en elastic material does not appear or disappear by itself but is only generated by longitudinal force exerted by the neighboring volume. It is interesting to note that compression and rarefaction occur alternatively.

Plastic regime. Viewing (29) as an equation of continuity, $G \boldsymbol{j}$ can be interpreted as a flow of charge $\rho \nabla \cdot \boldsymbol{v}$. Thus, we can put

$$
G \boldsymbol{j}=\boldsymbol{W}_{d} \rho \nabla \cdot \boldsymbol{v} .
$$

Here $\boldsymbol{W}_{d}$ is the drift velocity of the charge $\rho \nabla \cdot \boldsymbol{v}$ of the unit volume. As will be discussed below, if the charge is positive, it flows in the same direction as the local velocity of the material to dissipate the kinetic energy carried by the material particles. If it is negative, the charge flows in the direction opposite to the local velocity. Thus, we can put

$$
\boldsymbol{W}_{d}=\sigma_{0} \boldsymbol{v} .
$$

Here $\sigma_{0}$ is a dimensionless parameter that represents the degree of energy dissipation; the greater $\sigma_{0}$ is, the more energy is dissipated. With (38) and (39), (28) can be put in the form

$$
\begin{aligned}
\rho \frac{\partial \boldsymbol{v}}{\partial t} & =-G \nabla \times \boldsymbol{\omega}-\sigma_{0} \rho(\nabla \cdot \boldsymbol{v}) \boldsymbol{v} \\
& =-G \nabla \times \boldsymbol{\omega}-\sigma_{c} \boldsymbol{v} .
\end{aligned}
$$




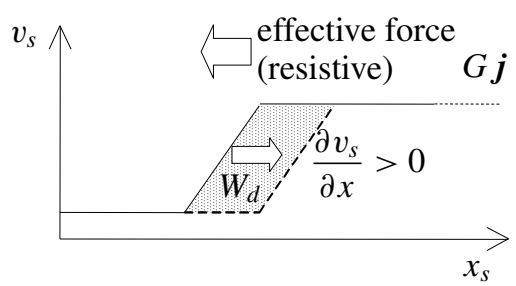

Figure 7. Positive charge flowing in the direction of local particle velocity.

The first term on the right-hand side of (40) represents recovery force due to shear deformation. Being proportional to the velocity, the second term can be interpreted as representing a velocity damping force, where

$$
\sigma_{c}=\sigma_{0} \rho(\nabla \cdot v)
$$

is the damping coefficient. This effect is interpreted as the energy-dissipative nature of plastic deformation.

Consider the physical meaning of the damping coefficient $\sigma_{c}$ and the dimensionless parameter that represents the degree of energy dissipation $\sigma_{0}$. To this end, rewrite the $\nabla \cdot \boldsymbol{v}$ that appears on the right-hand side of (41) as

$$
\begin{aligned}
\nabla \cdot \boldsymbol{v} & =\frac{\partial v_{x}}{\partial x}+\frac{\partial v_{y}}{\partial y}+\frac{\partial v_{z}}{\partial z} \\
& =\frac{\partial v_{s}}{\partial x} \alpha+\frac{\partial v_{s}}{\partial y} \beta+\frac{\partial v_{s}}{\partial z} \gamma \\
& =\frac{\partial v_{s}}{\partial x} \frac{d x}{d x_{s}}+\frac{\partial v_{s}}{\partial y} \frac{d y}{d x_{s}}+\frac{\partial v_{s}}{\partial z} \frac{d z}{d x_{s}}=\frac{d v_{s}}{d x_{s}} .
\end{aligned}
$$

Here $\alpha, \beta$ and $\gamma$ are direction cosines and $\boldsymbol{v}_{s}$ is the local velocity vector of the particle. With (39) and (42), we can put the plastic longitudinal force density (38) in the form

$$
G \boldsymbol{j}=\sigma_{0} \rho \frac{d v_{s}}{d x_{s}} v_{s} \hat{v}_{s} .
$$

Consider the right-hand side of (43) for the case $\sigma_{0}=1$. Since $v_{s}=d x_{s} / d t$, it can be put as

$$
G \boldsymbol{j}=\sigma_{0} \rho \frac{d v_{s}}{d x_{s}} v_{s} \hat{v}_{s}=\rho \frac{d v_{s}}{d x_{s}} \frac{d x_{s}}{d t} \hat{v}_{s}=\rho \frac{d v_{s}}{d t} \hat{v}_{s}=\frac{d}{d t}\left(\rho v_{s}\right) \hat{v}_{s} .
$$

The rightmost side of (44) represents the temporal change of momentum density (the momentum change of the unit volume). Thus, (43) can be interpreted as indicating the effect that the external longitudinal force has to cause the unit volume to gain momentum. In other words, when $\sigma_{0}=1$, the external force exerted by the neighboring volume contributes to the momentum increase of this unit volume without energy dissipation. From (38), we know that the charge drifts in the same 

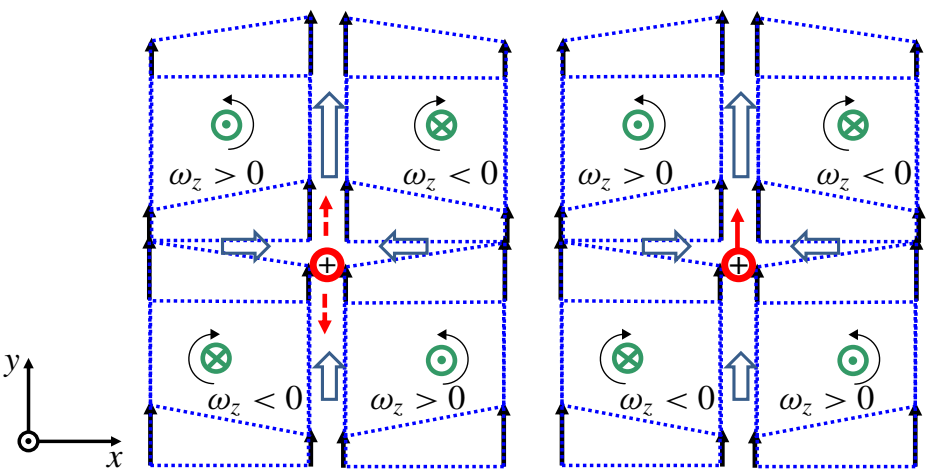

Figure 8. Schematic illustration of shear strain. Four blocks of the specimen under tensile load experience rotation-like behaviors due to differential vertical displacement indicated with narrow arrows at corners of blocks. Thicker arrows indicate resulting displacement along boundaries of the four blocks. The blocks' rotations generate tensile strain near the center of the specimen. Left: the elastic situation where the volumes neighboring this central volume at the top and bottom resist the tensile strain by exerting elastic force that can be viewed as the shear force represented by $\nabla \times \omega$. Right: the plastic situation where the material yields to the shear force and consequently the central volume drifts upward. This can be viewed as a positive charge drifts upward causing energy dissipation due to the friction exerted by surrounding materials.

direction as the longitudinal force at the drift velocity $\boldsymbol{W}_{d}$. From (43) when $\sigma_{0}=1$, $\boldsymbol{W}_{d}$ is equal to the particle velocity $\boldsymbol{v}$. So in this case, the charge $d v_{s} / d x_{s}$ drifts in the direction of the longitudinal force at the particle velocity $v_{s}$.

When $\sigma_{0}>1$, the situation is slightly different. In this case, as Figure 7 schematically illustrates, the drift velocity $W_{d}$ defined above can be interpreted as representing the motion of the pattern of $d v_{s} / d x_{s}$. Here the example shown in Figure 7 is a case where the particle velocity $v_{s}$ has positive gradient with respect to the coordinate axis $x_{s}$. Notice that, if $W_{d}>v_{s}$, the particles behind the leading edge of the pattern $d v_{s} / d x_{s}$ loses their momentum as the pattern passes because their velocity decreases. Here the rate of the momentum loss is $W_{d} \rho d v_{s} / d x_{s}$. From the viewpoint of the energy of the total system, this decrease in the momentum can be viewed as the reduction in the mechanical energy. The faster the motion of the pattern, the more energy the system loses; in the form of $W_{d}=\sigma_{0} v_{s}, \sigma_{0}>0$, the greater the value of $\sigma_{c}$, the greater the energy loss. For simplicity, the $x_{s}$ dependence of $v_{s}$ is assumed linear in Figure 7, but the same argument holds for any $x_{s}$ dependence as far as the pattern $d v_{s} / d x_{s}$ drifts in the direction of the longitudinal force $G \boldsymbol{j}$. 
The physical meaning of the dimensionless parameter $\sigma_{0}$ can be argued based on microscopic deformation dynamics. According to dislocation theory, plastic deformation occurs when dislocations propagate in the direction of shear stress. Here the driving force of the propagation is the local shear force, and these mobile dislocations are subject to intensive frictional force exerted by the surrounding atoms [Suzuki et al. 1991]. Based on the present field theory, this process can be explained with (28) and (40). In this context, the density on the left-hand side of these equations represents the mass of the unit volume experiencing shear strain. The first term on the right-hand side, $\nabla \times \omega$, represents the shear force that drives mobile dislocations. The second term represents the longitudinal force exerted on the unit volume. When the material responds to the shear force elastically, as Figure 8, left, illustrates schematically, this longitudinal force is elastic force exerted by the volume behind and in front of the unit volume along the line of shear. In this situation, the longitudinal force term $G \boldsymbol{j}$ on the right-hand side of (28) represents this elastic force. When the local material yields to the shear force and starts to be deformed plastically, defects (dislocations) are generated behind or in front of the unit volume and they propagate as the shear force $\nabla \times \omega$ is still effective. Figure 8, right, illustrates the situation schematically. In this case, the longitudinal force term $G \boldsymbol{j}$ takes the form of (40). As the defects are generated, the unit volume starts to drift and the dimensionless parameter $\sigma_{0}$ indicates how easily it drifts. The momentum loss discussed in Figure 7 can be interpreted as representing the energy-dissipative dynamics associated with the frictional force that the dislocations experience as they propagate. The rate of propagation of dislocations is a unique quantity of a given solid. Thus, it is natural to assume that the dimensionless parameter $\sigma_{0}$ is a material constant. It should be noted that the damping coefficient $\sigma_{c}$ depends on $\nabla \cdot \boldsymbol{v}$ according to (41) and varies as the deformation status changes. As will be discussed later, an increase in $\nabla \cdot v$ represents strain concentration and that increases the degree of energy dissipation.

Optical interferometric experiments performed on tensile-loaded metal-plate specimens [Yoshida et al. 1996; 1998] indicate that from time to time the interferometric fringe pattern shows a band structure as shown at the top of Figure 9. In this
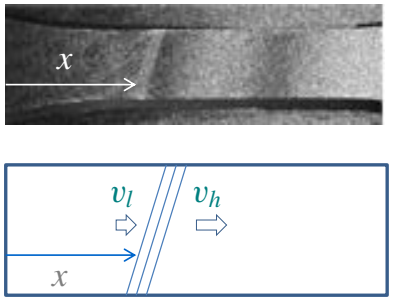
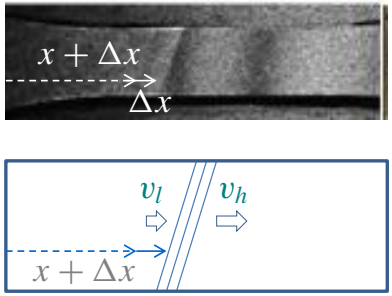

Figure 9. Developed, one-dimensional charge. 
type of optical interferometry, an interferometric image of the specimen is taken continuously with a constant interval. Here the interferometric image is formed by illuminating the specimen with a pair of laser beams so that they interfere with each other on the specimen surface in such a way that the relative optical phase difference is proportional to the displacement of interest. In the case of Figure 9, the interferometer is arranged to be sensitive to in-plane displacement parallel to the tensile axis. The image taken at each time step is subtracted from the image taken at a certain time step later. The result of the subtraction yields a fringe pattern that displays a whole-field map of the differential displacement that occurs between the two time steps. Since this differential displacement is proportional to the average velocity of the duration between the two time steps, this type of fringe pattern can be viewed as representing the velocity field. Thus, hereafter, the differential displacement field derived from the fringe pattern is referred to as the velocity field. Figure 9 is a sample fringe pattern. The dark stripes seen in Figure 9 are called the interferometric fringes that represent the contours of the velocity field. Each contour indicates that the velocity along the dark fringe corresponds to a relative phase change of an integer multiple of $2 \pi$. Often a band-structured concentrated fringe pattern appears and drifts along the length of the specimen as the bottom illustrations of Figure 9 indicate schematically. In specimens free of initial stress concentration, the banded structure typically starts to appear near the yield point. In specimens with initial stress concentration, the band structure can appear at any point before the specimen yields. The higher the degree of stress concentration, the earlier it appears. It is apparent that this band structure represents plastic deformation.

Based on the observation that it represents plastic deformation, this banded structure can be interpreted as a special case of the charge $\nabla \cdot \boldsymbol{v}=d v_{s} / d x_{s}$ discussed above where the velocity field depends only on the $x_{s}$ axis for the entire width of the specimen at the band-structured region. Figure 10 illustrates the situation schematically where a tensile load applied to a rectangular specimen generates a field of velocity vectors to the right and forms a pattern of $d v_{s} / d x_{s}>0$. The three parallel lines represent the pattern of $d v_{s} / d x_{s}$ where each line is a contour of constant velocity. The $x_{s}$ axis is perpendicular to the contours, and in accordance with the above argument, as the pattern drifts in the direction of $x_{s}$, the mechanical energy of the system is dissipated. The $x_{p}$ axis is set parallel to the velocity contour. As the velocity field does not depend on the $x_{p}$ axis $\left(d v_{p} / d x_{p}=0\right)$, we call this

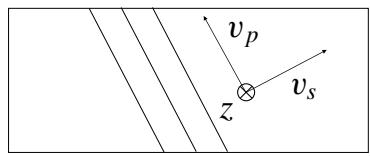

Figure 10. Developed, one-dimensional charge. 
type of pattern the one-dimensional charge. As the contours cross over the width of the specimen, we call it a developed charge. Thus, the pattern shown in Figure 10 is classified as a developed, one-dimensional charge.

A developed, one-dimensional charge is often observed in tensile experiments on a low-carbon steel at the beginning of the plastic regime where the stressstrain curve shows a plateau (the yield plateau). From the temporal behavior and other features, the one-dimensional charge observed in the yield plateau has been identified as representing the phenomenon known as the Lüders band [1860]. In aluminum-alloy specimens, similar band-like interferometric fringe patterns that can also be interpreted as a one-dimensional charge are often observed in a late stage of plastic deformation. Previous studies [Yoshida et al. 1996; 1998; Yoshida and Toyooka 2001] indicate that this type of pattern represent the shear band known as the Portevin-Le Chatelier (PLC) band. A number of optical interferometric experiments indicate that, if a one-dimensional charge appears in an early or a middle stage of plastic deformation, it moves around the specimen continuously; if it appears in a late stage of plastic deformation, it tends to appear intermittently and converge its movement to a certain place of the specimen where the specimen eventually fails. We call the former type the Lüders-band-like charge and the latter the PLC-band-like charge.

From experimental observation of a developed, one-dimensional charge, we can estimate the actual value of $\sigma_{0}$ as follows. A previous series of tensile experiments (personal communication with T. Sasaki, 2014) on an aluminum alloy indicate that the drift velocity of the Luiders-band-like charge is proportional to the cross-head speed. In this series of experiments, the cross-head speed was set at a constant rate for each test in a range of $0.1 \mathrm{~mm} / \mathrm{min}$ to $3.0 \mathrm{~mm} / \mathrm{min}$. Since only one Lüdersband-like charge appeared at a time and the number of fringes inside the charge was much greater than on the outside, we can say that the particle velocity inside the charge is approximately equal to the cross-head speed. Thus, we can approximate the magnitude of $\boldsymbol{v}$ appearing on the right-hand side of (39) $\left(W_{d}=\sigma_{0} v\right)$ by the crosshead speed; in other words, $\sigma_{0}$ is approximately equal to the constant of proportionality between the observed velocity of the Lüders-band-like charge $\left(W_{d}\right)$ and the cross-head speed $(\approx v)$. Based on this argument, the dimensionless parameter $\sigma_{0}$ can be estimated as $\sigma_{0} \approx 3000$.

Fracture regime. Fracture can be viewed as the final stage of plastic deformation. The transition from a late stage of plastic deformation to fracture can be conveniently discussed based on the longitudinal force $G \boldsymbol{j}$. With the use of the dimensionless parameter $\sigma_{0}$, the plastic longitudinal force expression (38) can be put in the form

$$
G \boldsymbol{j}=\boldsymbol{W}_{d} \rho \nabla \cdot \boldsymbol{v}=\sigma_{0} \rho(\nabla \cdot \boldsymbol{v}) \boldsymbol{v}
$$


A number of experiments indicate that, toward the end of plastic deformation, the one-dimensional charge decelerates and eventually becomes stationary at the location where the specimen fails [Yoshida et al. 1996; 1998]. The deceleration of the charge, or the corresponding decrease of the drift velocity $W_{d}$, can be understood as follows. Toward the end of the plastic regime, the material loses its capability of increasing the number of defects. In other words, the atomic arrangement does not have further room to create new dislocations. This situation eventually develops to the point where the value of $\sigma_{0}$ becomes zero. ${ }^{3}$ If the external force is still active, the material needs to dissipate the energy so that the total energy is conserved. In terms of the energy-dissipative force (45), $G \boldsymbol{j} \neq 0$ and $\boldsymbol{W}_{d}=0$. The only possibility to make this situation to happen is $\nabla \cdot v \rightarrow \infty$. This condition can be interpreted as representing that particles flow out of the unit volume at an infinitely high rate. Obviously such a condition causes the unit volume to be empty, or the material becomes discontinuous at this location. From the viewpoint of the gauge field, the system loses the charge of symmetry that connects (the charge of the connecting field) the material to be a continuum. This is the field-theoretical definition of fracture. The above-mentioned experimental observation that a onedimensional charge stops moving in a late stage of tensile experiment where the specimen eventually fails can be interpreted as experimental evidence of this idea about the fracture.

\section{Wave dynamics of deformation and supporting experiments}

3.1. Elastic compression wave. Substitution of (37) into (30) yields

$$
\left.\rho \frac{\partial^{2}(\nabla \cdot \boldsymbol{\xi})}{\partial t^{2}}=\nabla \cdot \nabla(\lambda+2 G)(\nabla \cdot \boldsymbol{\xi})=(\lambda+2 G) \nabla^{2}(\nabla \cdot \xi)\right) .
$$

This is the equation of an elastic compression wave traveling with phase velocity

$$
c_{\mathrm{comp}}=\sqrt{\frac{\lambda+2 G}{\rho}} .
$$

Next, replace $\boldsymbol{v}$ with $\boldsymbol{\xi}$, substitute (37) for $G \boldsymbol{j}$ in (28), and take the curl of the resultant equation:

$$
\rho \frac{\partial^{2}(\nabla \times \xi)}{\partial t^{2}}=-G \nabla \times \nabla \times \omega+\nabla \times \nabla(\lambda+2 G)(\nabla \cdot \xi) .
$$

With the mathematical identities $\nabla \times \nabla f=0$ where $f=(\lambda+2 G)(\nabla \cdot \xi)$ and

${ }^{3}$ As discussed above, $\sigma_{0}$ is a material constant. The fact that it becomes zero means that at this point the material is no longer the same as before. 
$\nabla \times \nabla \times \omega=\nabla(\nabla \cdot \omega)-\nabla^{2} \omega$, this leads to

$$
\rho \frac{\partial^{2} \boldsymbol{\omega}}{\partial t^{2}}=G \nabla^{2} \boldsymbol{\omega}
$$

Here $\nabla \cdot \boldsymbol{\omega}=0$ (see (26)) is used to find $\nabla(\nabla \cdot \boldsymbol{\omega})=0$. Equation (49) is the equation of an elastic rotational wave traveling with the phase velocity $c_{\text {shear }}$ (see (18)). These arguments show that the field equations (23)-(26) reduce to the elastic wave equations discussed in continuum mechanics. It is interesting to note that, when the force is proportional to the differential displacement in the form of $\nabla(\nabla \cdot \xi)$ (see (37)), the longitudinal term $G \boldsymbol{j}$ vanishes when we consider the rotational effect by taking the curl of the equation because of the mathematical identity $\nabla \times \nabla f=0$. This reflects the fact that the elastic force law is essentially longitudinal or orientation-preserving; longitudinal force does not contribute to rotational dynamics under the elastic force law.

3.2. Plastic transverse decaying wave. Elimination of $\boldsymbol{\omega}$ from (40) with the use of field equation (25) leads to the following wave equation that governs the velocity field $v$ :

$$
\rho \frac{\partial^{2} \boldsymbol{v}}{\partial t^{2}}-G \nabla^{2} \boldsymbol{v}+\sigma_{c} \frac{\partial \boldsymbol{v}}{\partial t}=-G \nabla(\nabla \cdot \boldsymbol{v}) .
$$

In the pure plastic regime where the longitudinal force $G \boldsymbol{j}$ is completely energydissipative (see (38) and (39)) and therefore the longitudinal restoring force mechanism is absent, (50) yields decaying transverse wave solutions. The right-hand side of (50) indicates that the spatial distribution of the deformation charge is the source term of this differential equation. When the charge is uniformly distributed over the entire specimen, $\nabla(\nabla \cdot v)=0$ and this differential equation becomes homogeneous. In this case, we can solve it analytically and express the solution in the form

$$
\boldsymbol{v}(t, \boldsymbol{r})=\boldsymbol{v}_{0} e^{-\left(\sigma_{c} / 2 \rho\right) t} \cos \left(\sqrt{\frac{G}{\rho} k^{2}-\frac{\sigma_{c}^{2}}{4 \rho^{2}}} t-\boldsymbol{k} \cdot \boldsymbol{r}\right) .
$$

Here $\boldsymbol{k}$ is the propagation vector.

Consider the physical meaning of the condition $\nabla(\nabla \cdot v)=0$. In an $x y z$ coordinate system, the condition can be put as

$$
\begin{aligned}
\frac{\partial}{\partial x}\left(\frac{\partial v_{x}}{\partial x}+\frac{\partial v_{y}}{\partial y}+\frac{\partial v_{z}}{\partial z}\right) & =\frac{\partial^{2} v_{x}}{\partial x^{2}}+\frac{\partial^{2} v_{y}}{\partial x \partial y}+\frac{\partial^{2} v_{z}}{\partial z \partial x}=0, \\
\frac{\partial}{\partial y}\left(\frac{\partial v_{x}}{\partial x}+\frac{\partial v_{y}}{\partial y}+\frac{\partial v_{z}}{\partial z}\right) & =\frac{\partial^{2} v_{x}}{\partial x \partial y}+\frac{\partial^{2} v_{y}}{\partial y^{2}}+\frac{\partial^{2} v_{z}}{\partial y \partial z}=0 \\
\frac{\partial}{\partial z}\left(\frac{\partial v_{x}}{\partial x}+\frac{\partial v_{y}}{\partial y}+\frac{\partial v_{z}}{\partial z}\right) & =\frac{\partial^{2} v_{x}}{\partial z \partial x}+\frac{\partial^{2} v_{y}}{\partial y \partial z}+\frac{\partial^{2} v_{z}}{\partial z^{2}}=0 .
\end{aligned}
$$


Equations (52)-(54) indicate that under this condition the velocity field is transversewave-like. Here is the explanation. As a sufficient condition for (52)-(54), we can set each of the nine terms appearing after the first equals sign of the three equations to zero. Under such a condition, the first column of this part indicates that the secondary derivative of $v_{x}$ is zero if the differentiation involves $\partial / \partial x$. This leads to the following generalized statements: the secondary derivative of $v_{i}$ is zero if the differentiation involves $\partial / \partial x_{i}$ and the only surviving nonzero secondary derivative takes the form of $\partial^{2} v_{i} / \partial x_{j}^{2}, i \neq j$. Note that these surviving secondary derivative terms come from the $\nabla^{2} \boldsymbol{v}$ term of the wave equation (50) and not from the source term. The transverse oscillatory dynamics is due to the shear mechanism represented by $\nabla \times \omega$. This argument indicates that the velocity wave in the pure plastic regime is essentially transverse. From this standpoint, we can put the solution (51) in the following form to express the transverse wave characteristic in a two-dimensional case. This will be compared to an experimental observation shortly below. Here $k_{y}$ is the $y$ component of $\boldsymbol{k}$, the interferometer has sensitivity in $y$, and the constant phase is omitted:

$$
v_{x}(t, \boldsymbol{r})=v_{x 0} e^{-\left(\sigma_{c} / 2 \rho\right) t} \cos \left(\sqrt{\frac{G}{\rho} k^{2}-\frac{\sigma_{c}^{2}}{4 \rho^{2}}} t-k_{y} y\right) .
$$

The wave solution (55) indicates that, if $\sigma_{c}$ is constant, the velocity field decays exponentially with the decay constant

$$
\tau_{c}=\frac{2 \rho}{\sigma_{c}}=\frac{2}{\sigma_{0}(\nabla \cdot v)} .
$$

Based on the above argument that the dimensionless parameter $\sigma_{0}$ is a materialdependent constant until the solid material fractures, we can estimate the charge density $(\nabla \cdot v)$ from (56) if $\sigma_{0}$ and the decay constant $\tau_{c}$ are known. As mentioned earlier in this paper, the damping coefficient $\sigma_{c}$ is proportional to the charge density (see (41)), and the higher the charge density, the more energy-dissipative the solid material is. Apparently, the one-dimensional, developed charge is a concentrated charge. In the context of tensile-loading, the differential velocity between the dynamic and static grips of the tensile machine is concentrated into the banded region that represents a developed charge. This situation is contrastive to an early stage of plastic deformation where the mechanical energy provided by the tensile machine is obviously dissipated via irreversible deformation but a developed charge is not present. It is interesting to estimate the charge density for both cases and compare them.

Figure 11 shows the decay characteristics of the velocity (differential displacement) field under a tensile experiment on an aluminum-alloy, thin-plate specimen. The tensile load was applied at a constant cross-head speed of $5.8 \times 10^{-6} \mathrm{~m} / \mathrm{s}$, and 

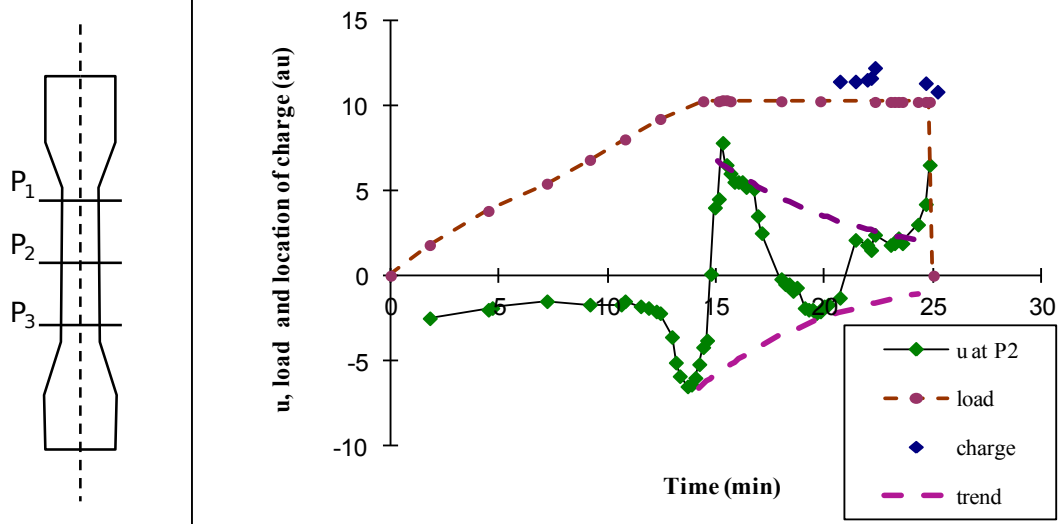

Figure 11. Decaying oscillation observed in a transverse plastic deformation wave.

the material was the same type as the above case where the dimensionless parameter $\sigma_{0}$ was estimated to be 3000 based on the proportionality between the drift velocity of the developed charge and the cross-head speed. The graph in Figure 11 plots the velocity vector component perpendicular to the tensile axis (the transverse velocity) evaluated at the reference point $P_{2}$ shown in the left margin of the figure. Also plotted are the load recorded by the tensile machine and the location of PLC-like charge that started to appear toward the end of the plastic regime. The horizontal axis is the time elapsed from the beginning of the tensile-loading. The oscillatory behavior of the transverse velocity starts near the yield point. In fact, observation of the transverse velocity at the other reference points $\left(P_{1}\right.$ and $\left.P_{3}\right)$ indicates that the oscillatory behavior propagates as a transverse wave [Yoshida et al. 1999]. Thus, it is reasonable to interpret the plot in Figure 11 as the decay characteristic of the transverse velocity wave in the plastic regime. Since the developed charge does not appear until the oscillatory behavior fades out, it is expected that the charge density is lower than that typically observed under the existence of a PLC-like charge.

From the trend of the oscillation peaks seen in Figure 11, the decay time constant of this case can be estimated as $6.7 \mathrm{~min}=400 \mathrm{~s}$. Substituting this value into the left-hand side of (56) and using $\sigma_{0}=3000$ for the aluminum-alloy case, we can estimate the charge density during this decay process as $(\nabla \cdot \boldsymbol{v})=2 /(400 \times 3000)=$ $1.7 \times 10^{-6} 1 / \mathrm{s}$. On the other hand, the charge density when the PLC-like charge appears can be estimated as follows. Using the same logic as above that the velocity of the leading edge (the edge closer to the dynamic grip) of the charge is approximately equal to that of the dynamic grip and the velocity of the tailing edge is zero, and using the band width of $5.2 \mathrm{~mm}$ along the tensile axis, we 
can evaluate the charge density $d v_{y} / d y=5.8 \times 10^{-6} \mathrm{~m} / \mathrm{s} / 5.2 \times 10^{-3} \mathrm{~mm}=$ $1.1 \times 10^{-3} 1 / \mathrm{s}$. As expected, this charge density is three orders of magnitude higher than $(\nabla \cdot v)=2 /(400 \times 3000)=1.7 \times 10^{-6} 1 / \mathrm{s}$ evaluated at the beginning of the oscillatory behavior of the transverse velocity.

3.3. Solitary wave in plastic regime. In the transitional stage from the elastic regime to the plastic regime, optical interferometric experiments often show a band-structured, interferometric fringe pattern that can be interpreted as the onedimensional charge expressed by (42) and illustrated by Figures 9 and 10. From the similarity in various behaviors, as mentioned above, this type of one-dimensional charge can be interpreted as representing the same phenomenon as the Lüders band [Yoshida et al. 2005]. Among these behaviors, the following two are interesting from the viewpoint of dynamics: (a) their drift velocity is proportional to the tensile rate and (b) the stress remains practically the same while they drift. Mertens et al. [1997] have made detailed analyses on dynamic behaviors of the Lüders band. They explain the mechanism of the phenomenon as the propagation of mobile dislocations at the plastic deformation front weakens the neighboring areas and the resultant deformation creates new dislocations. They also explain that the deformation at the front creates a strain jump that is roughly constant during the drift of the band and therefore its drift velocity is proportional to the pulling rate. Here an attempt is made to explain the behaviors (a) and (b) based on the present field theory.

Figure 10 illustrates schematically that one can characterize the velocity contours inside a one-deformation charge of this type as

$$
\begin{gathered}
\frac{\partial}{\partial x_{s}} \neq 0, \\
\frac{\partial}{\partial x_{p}}=0 .
\end{gathered}
$$

In this two-dimensional picture, the one-dimensional, developed charge is expressed as

$$
\nabla \cdot \boldsymbol{v}=\frac{\partial v_{p}}{\partial x_{p}}+\frac{\partial v_{s}}{\partial x_{s}}=\frac{d v_{s}}{d x_{s}}
$$

Similarly, the volume expansion appearing in the elastic longitudinal force expression (37) is

$$
\nabla \cdot \xi=\frac{\partial \xi_{p}}{\partial x_{p}}+\frac{\partial \xi_{s}}{\partial x_{s}}=\frac{d \xi_{s}}{d x_{s}}
$$

The rotation vector has only the $z$ component:

$$
\boldsymbol{\omega}=\omega_{z} \hat{z}=\left(\frac{\partial \xi_{s}}{\partial x_{p}}-\frac{\partial \xi_{p}}{\partial x_{s}}\right) \hat{z}
$$


Thereby, its rotation can be expressed as

$$
\nabla \times \boldsymbol{\omega}=\frac{\partial \omega_{z}}{\partial x_{s}} \hat{x}_{p}-\frac{\partial \omega_{z}}{\partial x_{p}} \hat{x}_{s}=\frac{\partial \omega_{z}}{\partial x_{s}} \hat{x}_{p},
$$

where the $z$ axis is perpendicular to the $x_{s}-x_{p}$ plane and condition (58) is used in going through the last equal sign.

Equations (59), (60) and (62) respectively indicate that inside the one-dimensional charge along the $x_{s}$ axis both the longitudinal plastic force proportional to $\nabla \cdot v$ and elastic force proportional to $\nabla \cdot \xi$ are potentially nonzero, and the shear force component $(G \nabla \times \omega)_{s}$ is zero. From the fact that the entire specimen is being pulled by the tensile machine, it is apparent that this elastic longitudinal force is tensile. The fact that the stress recorded by the tensile machine does not increase indicates that, as this tensile force stretches the banded region, stress drops occur presumably associated with the creation of new mobile dislocations. Thus, it is reasonable to assume that the elastic behavior is confined within the banded region. This argument leads to the following physical model. An elastic medium isolated to the location of the band moves in the entire specimen due to the longitudinal elastic force. The elastic dynamics is not transferred to outside of the banded region due to the plastic deformation associated with the creation of dislocations at the front. As the charge represented by the band region moves, the plastic longitudinal force causes energy dissipation. The coexistence of the elastic and plastic deformation makes total sense as this phenomenon takes place in the transitional stage from the elastic regime to the plastic regime.

Based on the above explanation, we can start a quantitative argument from the equation of motion of the elastic block (called the block) confined in the banded region. The net elastic force acting on the block is the differential force between the front and back surfaces of the block. At each surface, the elastic force is proportional to the local stretch, as Figure 12 illustrates schematically:

$$
\eta\left(x_{s}\right)=\frac{\partial \xi_{s}\left(x_{s}\right)}{\partial x_{s}} \delta x_{s} .
$$

Here $\eta\left(x_{s}\right)$ is the stretch at $x_{s}, \xi\left(x_{s}\right)$ is the displacement at the same point, and $\delta x_{s}$ is the infinitesimal width of the plane at $x_{s}$. The displacement of the block from its equilibrium position $X$ is the differential displacement of its front and back ends:

$$
X=\frac{\partial \eta}{\partial x_{s}} \Delta x_{s}=\frac{\partial}{\partial x_{s}}\left(\frac{\partial \xi_{s}}{\partial x_{s}} \delta x_{s}\right) \Delta x_{s}=\frac{\partial^{2} \xi_{s}}{\partial x_{s}^{2}}\left(\delta x_{s} \Delta x_{s}\right),
$$

where $\Delta x_{s}$ is the width of the block. The corresponding elastic energy is

$$
U=\frac{1}{2} k X^{2}=\frac{1}{2} k\left(\frac{\partial^{2} \xi_{s}}{\partial x_{s}^{2}}\right)^{2}\left(\delta x_{s} \Delta x_{s}\right)^{2}=\frac{S E}{2}\left(\frac{\partial^{2} \xi_{s}}{\partial x_{s}^{2}}\right)^{2} \delta x_{s}\left(\Delta x_{s}\right)^{2},
$$


$\eta\left(x_{s}\right)$ : displacement from equilibrium due to elasticity

$$
\eta(x)=\left.(\partial \xi / \partial x)\right|_{x} \delta x \quad \eta(x+\Delta x)=\left.(\partial \xi / \partial x)\right|_{x+\Delta x} \delta x
$$

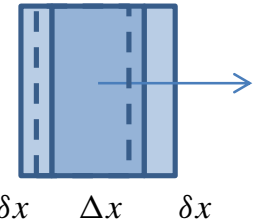

$X$ : band's displacement from equilibrium

$$
X=\frac{\partial \eta}{\partial x} \Delta x
$$

Figure 12. Elastic force acting on the isolated region that can be identified as a one-dimensional charge.

where $E$ is the elastic modulus associated with the longitudinal dynamics (corresponding to the stiffness or the Young's modulus in the elastic regime), ${ }^{4} S$ is the cross-sectional area and $k \delta x_{s}=S E$. This leads to the Lagrangian density of

$$
L_{\text {charge }}=\frac{U}{S \Delta x_{s}}=\frac{E}{2}\left(\frac{\partial^{2} \xi_{s}}{\partial x_{s}^{2}}\right)^{2}\left(\delta x_{s} \Delta x_{s}\right)=\frac{E}{2}\left(\partial_{x_{s}}^{2} \xi_{s}\right)^{2}\left(\delta x_{s} \Delta x_{s}\right) .
$$

Thus, the corresponding term of the Euler-Lagrangian equation of motion is

$$
\partial_{x_{s}}^{2}\left(\frac{\partial L_{\text {charge }}}{\partial\left(\partial_{x_{s}}^{2} \xi_{s}\right)}\right)=E \partial_{x_{s}}^{2}\left(\partial_{x_{s}}^{2} \xi_{s}\right)\left(\delta x_{s} \Delta x_{s}\right)=E \partial_{x_{s}}^{4} \xi_{s}\left(\delta x_{s} \Delta x_{s}\right) .
$$

Writing the traveling band in the form $\xi\left(x_{s}, t\right)=\xi\left(x_{s}-c_{w} t\right)$, we can replace one of the spatial derivatives with a temporal derivative by $\partial_{x_{s}}=-\partial_{t} \xi_{s} / c_{w}=-v_{s} / c_{w}$ (see (10)). With this, (67) becomes

$$
\partial_{x_{s}}^{2}\left(\frac{\partial L_{\text {charge }}}{\partial \partial_{x_{s}}^{2} \xi}\right)=-\frac{E}{c_{w}} \partial_{x_{s}}^{3}\left(\partial_{t} \xi_{s}\right)\left(\delta x_{s} \Delta x_{s}\right)
$$

When a developed charge appears, the material loses the elastic restoring force associated with $(\nabla \times \boldsymbol{\omega})_{s}$, and the longitudinal energy-dissipative force $\sigma_{0} \rho(\nabla \cdot \boldsymbol{v}) v=$ $\sigma_{0} \rho \partial v_{s} / \partial x_{s} v_{s}$ is active at the front where plastic deformation causes the stress drop. So (40) can be written in the form

$$
\rho \frac{\partial v_{s}}{\partial t}=-\sigma_{0} v_{s} \rho \frac{\partial v_{s}}{\partial x_{s}}-\frac{E \delta x_{s} \Delta x_{s}}{c_{w}} \partial_{x_{s}}^{3}\left(\partial_{t} \xi_{s}\right),
$$

${ }^{4} E$ is the stiffness associated with the normal stress or the longitudinal effect. In the plastic regime, or when the defect density is substantial, this value becomes lower than in the elastic regime. In the present context, it should be differentiated from the Young's modulus of the elastic regime. 
which can further be rewritten in a form more familiar as the Korteweg-de Vries equation [Maugin 2011]:

$$
\partial_{t} v+\sigma_{0} v \partial_{x} v+\frac{E \delta x_{s} \Delta x_{s}}{\rho c_{w}} \partial_{x}^{3} v=0 .
$$

Here, for clarity, the subscript $s$ has been omitted from the variables. As is well known, (70) yields the form of solution

$$
v(x, t)=a \operatorname{sech}^{2}\left(b\left(x-c_{w} t\right)\right),
$$

where

$$
\begin{aligned}
c_{w} & =\frac{\sigma_{0} a}{3}, \\
b^{2} & =\left(\frac{\sigma_{0} a}{3}\right)^{2} \frac{\rho}{4 E \delta x_{s} \Delta x_{s}} .
\end{aligned}
$$

In condition (72), $a$ is the amplitude of the velocity wave $v(x, t)$. It is reasonable to consider that this amplitude is proportional to the pulling rate of the specimen. This explains why the one-dimensional charge, and hence the Lüders band, drifts at a velocity proportional to the pulling rate. Condition (73) indicates that the width of the banded region is proportional to the square root of the elastic modulus $E$. This indicates that, as the material loses its elasticity with the development of plastic deformation, the one-dimensional charge tends to be narrower. This is consistent with experimental observations [Yoshida et al. 1996; 1998] and can be interpreted as the degree of stress concentration increasing with the development of plastic deformation.

\section{Conclusions}

Based on the field-theoretical approach associated with the local symmetry of linear elastic law, the dynamics of deformation and fracture has been discussed. Various conventionally known phenomena of deformation have been explained from the field-theoretical viewpoint. The concept of deformation charge has been introduced based on the analogy to electrodynamics and used to explain the energy-dissipative nature of plastic deformation. Decaying transverse wave characteristics of plastic deformation and solitary wave characteristics of the transitional stage from the elastic regime to the plastic regime have been discussed and compared to experimental results.

\section{References}

[Aitchison and Hey 1989] I. J. R. Aitchison and A. J. G. Hey, Gauge theories in particle physics, 2nd ed., Institute of Physics, Bristol, 1989. 
[Auffray et al. 2015] N. Auffray, F. dell'Isola, V. A. Eremeyev, A. Madeo, and G. Rosi, "Analytical continuum mechanics à la Hamilton-Piola least action principle for second gradient continua and capillary fluids", Math. Mech. Solids 20:4 (2015), 375-417.

[Fleck and Hutchinson 1997] N. A. Fleck and J. W. Hutchinson, "Strain gradient plasticity", Adv. Appl. Mech. 33 (1997), 295-361.

[Griffith 1921] A. A. Griffith, "The phenomena of rupture and flow in solids", Philos. T. Roy. Soc. A 221:587 (1921), 163-198.

[Hill 1998] R. Hill, The mathematical theory of plasticity, Oxford University, 1998.

[Ichinose et al. 2006] K. Ichinose, S. Yoshida, K. Gomi, K. Taniuchi, K. Fukuda, and H. Ishii, "Detection of crack initiation by observations of free surface condition", J. ASTM Int. 3:6 (2006), JAI13189.

[Irwin 1948] G. R. Irwin, "Fracture dynamics", pp. 147-166 in Fracturing of metals, edited by F. Jonassen et al., American Society for Metals, Cleveland, 1948.

[Javili et al. 2013] A. Javili, F. dell'Isola, and P. Steinmann, "Geometrically nonlinear higher-gradient elasticity with energetic boundaries", J. Mech. Phys. Solids 61:12 (2013), 2381-2401.

[Landau and Lifshitz 1986] L. D. Landau and E. M. Lifshitz, Course of theoretical physics, VII: Theory of elasticity, 3rd ed., Butterworth-Heinemann, Oxford, 1986.

[Lubliner 2008] J. Lubliner, Plasticity theory, Dover, Mineola, NY, 2008.

[Lüders 1860] W. Lüders, "Ueber die Aeußerung der Elasticität an stahlartigen Eisenstäben und Stahlstäben, und über eine beim Biegen solcher Stäbe beobachtete Molecularbewegung", Polytech. J. Dingler 155:8 (1860), 18-22.

[Madeo et al. 2013] A. Madeo, F. dell'Isola, and F. Darve, "A continuum model for deformable, second gradient porous media partially saturated with compressible fluids", J. Mech. Phys. Solids 61:11 (2013), 2196-2211.

[Maugin 2011] G. A. Maugin, "Solitons in elastic solids (1938-2010)", Mech. Res. Commun. 38:5 (2011), 341-349.

[Mertens et al. 1997] F. Mertens, S. V. Franklin, and M. Marder, "Dynamics of plastic deformation fronts in an aluminum alloy”, Phys. Rev. Lett. 78:23 (1997), 4502-4505.

[Mindlin 1965] R. D. Mindlin, "Second gradient of strain and surface-tension in linear elasticity", Int. J. Solids Struct. 1:4 (1965), 417-438.

[Spencer 1980] A. J. M. Spencer, Continuum mechanics, Longman, London, 1980.

[Suzuki et al. 1991] T. Suzuki, S. Takeuchi, and H. Yoshinaga, Dislocation dynamics and plasticity, Series in Materials Science 12, Springer, Berlin, 1991.

[Toupin 1964] R. A. Toupin, "Theories of elasticity with couple-stress", Arch. Rational Mech. Anal. 17:2 (1964), 85-112.

[Yoshida 2000] S. Yoshida, "Consideration on fracture of solid-state materials", Phys. Lett. A 270:6 (2000), 320-325.

[Yoshida 2008] S. Yoshida, "Dynamics of plastic deformation based on restoring and energy dissipative mechanisms in plasticity", Phys. Mesomech. 11:3-4 (2008), 137-143.

[Yoshida 2011] S. Yoshida, "Scale-independent approach to deformation and fracture of solid-state materials", J. Strain Anal. Eng. 46:5 (2011), 380-388.

[Yoshida 2015] S. Yoshida, Deformation and fracture of solid-state materials: field theoretical approach and engineering applications, Springer, New York, 2015. 
[Yoshida and Toyooka 2001] S. Yoshida and S. Toyooka, "Field theoretical interpretation on dynamics of plastic deformation: Portevin-Le Chatelie effect and propagation of shear band", J. Phys. Condens. Mat. 13:31 (2001), 6741-6757.

[Yoshida et al. 1996] S. Yoshida, Suprapedi, R. Widiastuti, M. Pardede, S. Hutagalong, J. S. Marpaung, A. F. Muhardy, and A. Kusnowo, "Direct observation of developed plastic deformation and its application to nondestructive testing”, Jpn. J. Appl. Phys. 35:7A (1996), L854-L857.

[Yoshida et al. 1998] S. Yoshida, Muchiar, I. Muhamad, R. Widiastuti, and A. Kusnowo, "Optical interferometric technique for deformation analysis", Opt. Express 2:13 (1998), 516-530.

[Yoshida et al. 1999] S. Yoshida, B. Siahaan, M. H. Pardede, N. Sijabat, H. Simangunsong, T. Simbolon, and A. Kusnowo, "Observation of plastic deformation wave in a tensile-loaded aluminumalloy”, Phys. Lett. A 251:1 (1999), 54-60.

[Yoshida et al. 2005] S. Yoshida, H. Ishii, K. Ichinose, K. Gomi, and K. Taniuchi, "An optical interferometric band as an indicator of plastic deformation front", J. Appl. Mech. 72:5 (2005), 792794.

Received 6 Feb 2015. Revised 24 May 2015. Accepted 18 Jul 2015.

SANICHIRO YoshidA: syoshida@selu.edu

Department of Chemistry and Physics, Southeastern Louisiana University, Hammond, LA 70402, United States 
EDITORIAL BOARD

ANTONIO CARCATERRA

ERIC A. CARLEN

FRANCESCO DELL'ISOLA

RAFFAELE ESPOSITO

ALBERT FANNJIANG

Gilles A. FranCFORT

Pierangelo MARCATI

JEAN-JACQUES MARIGO

PETER A. MARKOWICH

MARTIN OSTOJA-STARZEWSKI

PIERRE SEPPECHER

DAVID J. STEIGMANN

PAUl STEINMANN

PierRe M. SuQueT

MANAGING EDITORS

MICOL AMAR

CORRADO LATTANZIO

ANGELA MADEO

MARTIN OSTOJA-STARZEWSKI

ADVISORY BOARD

ADNAN AKAY

Holm AltenBaCH

MICOL AMAR

HARM ASKES

TEODOR ATANACKOVIĆ

VICTOR BERDICHEVSKY

GUY BOUCHITTÉ

ANDREA BRAIDES

ROBERTO CAMASSA

MAURO CARFORE

ERIC DARVE

FELIX DARVE

ANNA DE MASI

GianPiEtro DEL Piero

EMMANUELE Di BENEDETTO

BERNOLD FIEDLER

IRENE M. GAMBA

DAVID Y. GAO

SERGEY GAVRILYUK

TIMOTHY J. HEALEY

DOMINIQUE JEULIN

ROGER E. KHAYAT

CORRADO LATTANZIO

ROBERT P. LIPTON

ANGELO LUONGO

ANGELA MADEO

JUAN J. MANFREDI

CARLO MARCHIORO

GÉRARD A. MAUGIN

ROBERTO NATALINI PATRIZIO NEFF

ANDREY PIATNITSKI

ERRICO PRESUTTI

MARIO PULVIRENTI

LUCIO RUSSO

Miguel A. F. SANJUAN

PATRICK SElVADURAI

ALEXANDER P. SEYRANIAN

MIROSLAV ŠILHAVÝ

GUIDO SWEERS

ANTOINETTE TORDESILLAS

LEV TRUSKINOVSKY

JUAN J. L. VELÁZQUEZ VINCENZO VESPRI ANGELO VULPIANI msp.org/memocs

Università di Roma "La Sapienza", Italia

Rutgers University, USA

(CO-CHAIR) Università di Roma "La Sapienza", Italia

(TREASURER) Università dell'Aquila, Italia

University of California at Davis, USA

(CO-CHAIR) Université Paris-Nord, France

Università dell'Aquila, Italy

École Polytechnique, France

DAMTP Cambridge, UK, and University of Vienna, Austria

(CHAIR MANAGING EDITOR) Univ. of Illinois at Urbana-Champaign, USA

Université du Sud Toulon-Var, France

University of California at Berkeley, USA

Universität Erlangen-Nürnberg, Germany

LMA CNRS Marseille, France

Università di Roma "La Sapienza", Italia

Università dell'Aquila, Italy

Université de Lyon-INSA (Institut National des Sciences Appliquées), France

(CHAIR MANAGING EDITOR) Univ. of Illinois at Urbana-Champaign, USA

Carnegie Mellon University, USA, and Bilkent University, Turkey

Otto-von-Guericke-Universität Magdeburg, Germany

Università di Roma "La Sapienza", Italia

University of Sheffield, UK

University of Novi Sad, Serbia

Wayne State University, USA

Université du Sud Toulon-Var, France

Università di Roma Tor Vergata, Italia

University of North Carolina at Chapel Hill, USA

Università di Pavia, Italia

Stanford University, USA

Institut Polytechnique de Grenoble, France

Università dell'Aquila, Italia

Università di Ferrara and International Research Center MEMOCS, Italia

Vanderbilt University, USA

Freie Universität Berlin, Germany

University of Texas at Austin, USA

Federation University and Australian National University, Australia

Université Aix-Marseille, France

Cornell University, USA

École des Mines, France

University of Western Ontario, Canada

Università dell' Aquila, Italy

Louisiana State University, USA

Università dell'Aquila, Italia

Université de Lyon-INSA (Institut National des Sciences Appliquées), France University of Pittsburgh, USA

Università di Roma "La Sapienza”, Italia

Université Paris VI, France

Istituto per le Applicazioni del Calcolo "M. Picone", Italy

Universität Duisburg-Essen, Germany

Narvik University College, Norway, Russia

Università di Roma Tor Vergata, Italy

Università di Roma "La Sapienza”, Italia

Università di Roma “Tor Vergata”, Italia

Universidad Rey Juan Carlos, Madrid, Spain

McGill University, Canada

Moscow State Lomonosov University, Russia

Academy of Sciences of the Czech Republic

Universität zu Köln, Germany

University of Melbourne, Australia

École Polytechnique, France

Bonn University, Germany

Università di Firenze, Italia

Università di Roma La Sapienza, Italia

MEMOCS (ISSN 2325-3444 electronic, 2326-7186 printed) is a journal of the International Research Center for the Mathematics and Mechanics of Complex Systems at the Università dell'Aquila, Italy.

Cover image: "Tangle” by $\odot$ John Horigan; produced using the Context Free program (contextfreeart.org).

PUBLISHED BY

7 mathematical sciences publishers

nonprofit scientific publishing

http://msp.org/

(C) 2015 Mathematical Sciences Publishers 
Mathematics and Mechanics of Complex Systems vol. 3 no. 3

Stationary solutions of Keller-Segel-type crowd motion and 211 herding models: Multiplicity and dynamical stability

Jean Dolbeault, Gaspard Jankowiak and Peter

Markowich

Comprehensive description of deformation of solids as 243 wave dynamics

\section{Sanichiro Yoshida}

On the constitutive equations of viscoelastic micropolar 273 plates and shells of differential type

Holm Altenbach and Victor A. Eremeyev

Identification of higher-order elastic constants for grain 285 assemblies based upon granular micromechanics

Anil Misra and Payam Poorsolhjouy

MEMOCS is a journal of the International Research Center for the Mathematics and Mechanics of Complex Systems at the Università dell' Aquila, Italy.

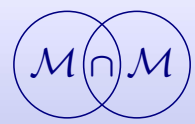

